\title{
Nomothetic Explanation and Humeanism about Laws of
}

\section{NATURE ${ }^{*}$}

\author{
Harjit Bhogal \\ Penultimate Version - Final Version forthcoming in Oxford Studies in Metaphysics
}

Imagine the mosaic of events that occur in this world, spread out across the totality of space and time. Within this mosaic, certain patterns are discernible. The core idea of Humeanism about laws of nature is that laws of nature are just patterns, or ways of describing patterns, in this mosaic. Laws of nature are not entities that stand above the mosaic, governing the patterns of events.

More precisely, take Humeanism about laws of nature to be the view that the laws of nature reduce to the Humean Mosaic — that is, the intrinsic physical state of each spacetime point (or each pointlike object) and the spatio-temporal relations between those points — and that the Humean Mosaic is not further reduced to anything else. (From now on I'll refer to the view simply as 'Humeanism'.)

Humeanism is a popular view, but currently existing versions face serious problems. In particular, there are a series of powerful objections to existing versions of the view.

Many of these objections share a guiding thought though; they are based on the idea that there is a certain kind of divergence between the practice of science and the metaphysical picture suggested by

\footnotetext{
*Thanks to Karen Bennett, Thomas Blanchard, Eddy Keming Chen, Anthony Dardis, Marco Dees, Tom Donaldson, Cian Dorr, Chris Dorst, Alison Fernandes, Kit Fine, Martin Glazier, Laura Franklin-Hall, Michael Townsen Hicks, David Kovacs, Barry Loewer, Tim Maudlin, Michaela McSweeney, Zee R Perry, Erica Shumener, Michael Strevens, Trevor Teitel, Dan Waxman, Mike Zhao and audiences at the Midsummer Philosophy Workshop, the Society for the Metaphysics of Science Annual Conference, and NYU Thesis prep for extremely helpful feedback and discussion.
} 
Humeanism. In particular, I'm going to focus on three such objections: non-supervenience objection, the non-fundamentality objection, and the explanatory circularity objection.1

I'm going to suggest, however, that this divergence is something that the Humean can make sense of. Implicit in a certain strand of Humean thinking, I claim, is the idea that some of the aims of science differ from the aims of metaphysics. For example, a certain type of Humean might naturally think that scientific explanation aims to unify in a way that metaphysical explanation does not.

Because of this, I claim, the Humean can argue that it is appropriate for there to be a divergence between certain elements of metaphysical and scientific practice. I develop this thought by sketching a novel version of Humeanism which accepts this kind of divergence and arguing that this version deals with the objections better than traditional Humean accounts.

And further, I argue that my approach can help the Humean give better accounts of other physical modalities - like counterfactuals, chance, and physical possibility - than they have been able to give so far.

Clearly, doing this all in the same paper is very ambitious, perhaps to an extent that's ill-advised. But it is important, I think, to see that many of the objections that Humeanism faces are closely related to each other and consequently, we can deal with the objections in a unified way. I'm not giving piecemeal responses to the objections that the Humean faces. Rather, I'm going to motivate and sketch a single Humean picture that deals with the objections together. So there is value in discussing them all in the same place.

This does come at a cost though. Since I'm dealing with multiple issues for the Humean which have traditionally been discussed separately I won't be able to discuss each issue as deeply. And I certainly won't be able to fully discuss all the literature on these issues. Rather, the aim is just to sketch a certain

\footnotetext{
${ }^{1}$ One common objection to Humeanism that I won't be considering is about the consistency of the view with quantum mechanical entanglement phenomena (see, for example, Maudlin (2007, pp. 50-61)). Some recent work has show how Humeanism need not be in conflict with quantum mechanics (see Bhogal and Perry (2017), Miller (2014), Esfeld et al. (2014)).
} 
Humean picture and show that this picture has promise for dealing with the objections to Humeanism. In section 1 I describe the three objections to Humeanism that I'm focusing on.

In section 2 I argue that the Humean, or at least a certain type of Humean, should expect a divergence between scientific and metaphysical practice and that seeing this allows us to respond to the objections discussed in section 1. This comes in two steps. Firstly, I develop Loewer's (2012) suggestion of distinguishing between scientific and metaphysical explanation, stressing the different aims of scientific and metaphysical explanation. And secondly, I extend this to a divergence between scientific and metaphysical notions of fundamentality and possibility.

In section $3 \mathrm{I}$ argue that this distinction between scientific and metaphysical possibility feeds through to other scientific notions. In particular, recognizing this different space of possibility motivates certain approaches to counterfactuals, physical possibility and chance.

\section{Problems for Humeanism}

In this section I'm going to briefly describe three objections to Humeanism and quickly look at some of the responses that are common in the Humean literature. As will become clear, these objections are all driven by the idea that there is a divergence between scientific practice and the practice of Humean metaphysics.

\subsection{Non-SuperVenience}

The most common objection to Humeanism is the non-supervenience objection. Humeanism implies that the laws supervene upon the Humean Mosaic. But this appears to be false; there appear to be worlds that match in their mosaics but differ in their laws.

There are many putative examples of this - see, for example Tooley (1977, p. 669) and Carroll (1994, 
pp. 57-67) - but here is a particularly simple one due to Maudlin (2007, pp. 67-68): Consider a universe that just consists in an empty Minkowski spacetime. Such a spacetime is a model of General Relativistic laws. 2 So it appears that there could be a world like this where the laws are General Relativistic. But, such a spacetime is also consistent with Special Relativity being the full story about spacetime and there being a different set of laws about gravitation. So it appears that there could be a world like this where Special Relativity is true and there is some non-General-Relativistic set of laws. But now we have two worlds that match in their mosaics (both consist in an empty Minkowski spacetime) but differ in their laws. Call these two worlds the empty-SR world and the empty-GR world

There are a couple of distinct ways of pressing this objection, which are not often distinguished in the literature. The first version of the objection is that Humeanism says false things about metaphysical possibility. It says that Humeanism implies that at least one of the two worlds described above is metaphysically impossible, but that is not true, both are metaphysically possible.

The second version of the objection is that Humeanism does not do justice to the scientific practice of reasoning about these cases. When scientists reason about GR and SR the two empty universe cases are both taken seriously and are treated as distinct. In fact, there look to be substantial differences between the cases. For example, there are counterfactuals that differ in truth-value between the worlds - in the empty-GR world if there was a massive object then spacetime would be curved. This is because the laws of General Relativity associate masses with curvature of spacetime. Not so in the empty-SR world. Explanations differ between the worlds too. For example, the (lack of) curvature of regions of spacetime is explained differently in the empty-GR world and the empty-SR world. The objection is that the Humean cannot make sense of how these cases are treated differently in scientific practice because, according to them, there are not two distinct worlds here which both have the same mosaic but differ in the laws, rather there is only one world with that mosaic.

\footnotetext{
${ }^{2}$ That is, the truth of the propositions that are the laws of General Relativity are consistent with an empty Minkowski spacetime.
} 


\subsection{NON-FUNDAMENTALITY}

In his (2007, p. 105) Maudlin objects to Humeanism as follows:

$[\mathrm{N}]$ othing in scientific practice suggests that one ought to try to reduce fundamental laws to anything else. Physicists simply postulate fundamental laws, then try to figure out how to test their theories; they nowhere even attempt to analyze those laws in terms of patterns of instantiation of physical quantities. The practice of science, I suggest, takes fundamental laws of nature as further unanalyzable primitives. As philosophers, I think we can do no better than to follow this lead.

One way (though not the only way) of understanding this passage is as an objection that Humeanism does not respect the practice of science. Scientists don't attempt to reduce away (certain) laws, rather they take them as fundamental. The Humean, however, does not take laws as fundamental. So Humeanism conflicts with scientific practice over what is fundamental. Call this the non-fundamentality objection.

\subsection{Explanatory Circularity}

Laws can explain particular events; specifically, they can explain features of the Humean Mosaic. But, the explanatory circularity objection says, Humeanism explains what the laws are in terms of the Mosaic. So we have a circularity.

For definiteness, I'm going to assume in this paper that this explanation of the laws in terms of the mosaic goes by way of a version of the Best System account of laws (see Lewis, 1983, p. 42-3). This is the most popular Humean view of laws. On this account the laws are given by systemizing all of

\footnotetext{
${ }^{3}$ Perhaps you don't find this objection pressing because it seems clear that the sense of fundamentality relevant to the scientist is not the same as the philosophical sense relevant to the debate over Humeanism. Such a thought turns out in my favor, since I will end up encouraging you to have similar thoughts with respect to explanation and possibility.
} 
the facts about the mosaic in a way that best balances simplicity and informativeness. Slightly more precisely, consider sets of axioms and the deductive closure of those axioms. Some set of axioms are informative about the mosaic - their deductive closure tells us a lot about the facts about the mosaics. Some sets of axioms are simple, in the sense of being syntactically simple when written down - there are few axioms and they are syntactically short. The axioms that best balance simplicity and informativeness best systematize the mosaic. The axioms of the best system (or some privileged subset thereof) count as the laws. ${ }^{-1}$

So, the Humean has to accept a certain kind of explanatory circularity — the laws explain particular events, and in turn those particular events partially explain the laws. But explanations cannot be circular in this way, so Humeanism is false.

Versions of this objection are also stated by Armstrong (1983, p. 40), Bird (2007, p. 86), Maudlin (2007, p. 172), and Hajek (2010, pp. 219-220), among many others.

\subsection{Responses to the Problems}

Humeans have had disappointingly little to say about the non-supervenience objection. Commonly Humeans have just bitten the bullet and accepted that it is not the case that both the empty-GR world and the empty-SR world are possible (see Earman and Roberts (2005, section 2), Loewer (1996, pp.192194), Roberts (1998, p. 428-433), Beebee (2000, sections 5-6)). 1 In fact, the typical move is to claim that neither are possible. Biting the bullet seems reasonable enough as a response to the first version of the objection - rejecting an intuition about metaphysical possibility is perhaps not too big a loss for the theory, especially given that all the authors cited above go on to argue that we shouldn't be confident in the reliability of such intuitions in this context.

\footnotetext{
${ }^{4}$ There are lots of further issues here, for example, about what language the axioms can be formulated in, about what exactly 'informativeness' means, and about how to weigh informativeness and simplicity. But these don't matter for our aims.

${ }^{5}$ Roberts (2008, pp. 358-361) is an exception to this. In effect, he claims that the empty-SR and empty-GR worlds exist and are the same world. This is possible because he has a very non-standard conception of laws - he calls it the meta-theoretic conception. I'm going to ignore this response and focus on the mainstream conception of law.
} 
But this doesn't help with the second version of the objection. The Humean still has to make sense of how, in our scientific reasoning, we recognize these two cases as distinct and says very different things - for example with respect to explanations and counterfactuals - about them. It seems plainly true that the empty-SR world and the empty-GR world differ, for example, in their explanations of the lack of curvature of spacetime. In the empty-GR world spacetime is not curved because there happen to be no massive objects in the world; in the empty-SR world spacetime is not curved because the basic geometry of spacetime does not allow it. It doesn't seem open for the Humean to reject this in the way they rejected the intuitions about metaphysical possibility. The Humean literature has been largely silent about such cases. But without a response, this is an extremely serious objection to Humeanism. Responses to the non-fundamentality objection are not common either. Though this is more understandable than the lack of response to the non-supervenience objection, given the relative rate at which the objections are pressed in the literature.

There has been more progress with respect to the explanatory circularity problem. In particular, Loewer (2012) has influentially suggested a response based on distinguishing 'scientific' and 'metaphysical' explanation. In the next section I'm going to develop this response and show how it is motivated from a certain Humean way of looking at the world. Further, I'm going to suggest that we can leverage this response into responses to the non-fundamentality and non-supervenience objections.

\section{The VIEW}

The objections of the previous section all point to a divergence between the practice of science and that of Humean metaphysics. The explanatory circularity objection says that scientific explanations run contrary to the explanations given by Humeanism. The non-fundamentality objection says that

\footnotetext{
${ }^{6}$ Another approach to the circularity problem which is gaining in popularity says that laws don't actually explain events in the mosaic, rather they explain why one event explains another (Skow, 2016; Hicks, ms). This response deserves much more attention that I can give it here, but it's rather unclear whether the view does, in fact, avoid problematic circularity (see, Lange (2013, 2018); Hicks (ms)).
} 
Humeanism disagrees with scientific practice about what is fundamental. The non-supervenience objection says that scientific practice regards as possible situations that Humeanism does not.

My strategy is not to deny this divergence, but to suggest that it is appropriate. In particular, the Humean should accept a distinction between the scientific and the metaphysical notions of explanation, fundamentality and possibility. Each of these three distinctions can be used to respond to one of the three problems discussed above. Ultimately, these distinctions are motivated by a Humean view where there are differing aims of science and of metaphysics.

\subsection{Explanatory Circularity and Two Types of Explanation}

Loewer (2012) suggests, but does not fully develop, a response to the explanatory circularity objection. The idea is to accept both that the laws explain elements of the mosaic and those elements explain the laws but to avoid circularity by claiming that there are two different senses of 'explain' - the mosaic metaphysically explains the laws, but the laws scientifically explain the mosaic. Loewer's terminology is apt to cause confusion though, because, as we will see, some metaphysical explanations are relevant to science. So, instead of distinguishing metaphysical and scientific explanation I'm going to distinguish metaphysical and nomothetic explanation.

In this section I will consider how the Humean can use this distinction to respond to the circularity objection and I'll discuss how this response is motivated from within the Humean picture. This response doesn't come for free; it requires commitments about the nature of nomothetic and metaphysical explanation. But these commitments naturally flow from the Humean viewpoint.

(One quick clarification: In what follows, I take explanation to be a 'mind-independent' relation between facts - one that holds in the world and is, in general, not determined by us. This is to be sharply distinguished from an act of explanation, which is typically a speech act.)

\footnotetext{
${ }^{7}$ Sometimes, for simplicity, I'll talk about events being explained. Strictly I mean that the fact that the event occurs is explained.
} 
Let's start by considering the distinction between nomothetic and metaphysical explanation. What is the difference between them? Consider nomothetic explanation first. I'm taking nomothetic explanation to be a type of scientific explanation - they are those where the laws of nature play an explanatory role. For example, an explanation of the velocity of a billiard ball from the facts about its recent collision and the Newtonian laws would count as a nomothetic explanation (if Newtonian mechanics were true). There may be other types of scientific explanation too, like pure causal explanations where the laws do not play an explanatory role. But I'm restricting my attention to nomothetic explanation because the circularity issue is about the laws of nature - whether laws can explain without circularity.

We have good reason, independent of Humeanism, to think that a relation of metaphysical explanation exists and is different from nomothetic explanation. In particular, many authors recently have noted that an asymmetric relation of metaphysical explanation is central to philosophical theorizing in many areas (for example, Fine (2001), Schaffer (2009), Rosen (2010)). This is the relation that holds between the fact that there is a table and the fact that there are atoms arranged tablewise (if certain views about composition are true); between the facts about mental states and the facts about brain states (if certain kinds of physicalism about mind are true); and between moral facts and the facts about God's commands (if certain kinds of divine command theory are true).

This type of explanation has some distinctive features. It is very closely connected to metaphysical dependence relations, like grounding or constitution. (Perhaps those relations 'back' metaphysical explanations, or perhaps those relations just are metaphysical explanations. Further, the explanandum in a metaphysical explanation is nothing over and above the explanans, or at least, the explanandum is, in some sense, not a substantial addition to the ontology when we already have the explanans. (For more substantial discussion, see Bennett (2017, Section 8.2.2) and Schaffer (2015).) Further, as Loewer (2012, p. 131) notes it looks like the relation of metaphysical explanation cannot be probabilistic.

Clearly, there are nomothetic explanations which aren't metaphysical explanations. So the relations of nomothetic and metaphysical explanation are not identical. 
Notice, that given the distinction between metaphysical and nomothetic explanation, the thesis of Humeanism - that laws are reduced to the Humean mosaic — is naturally precisified in terms of metaphysical explanation. In particular, we can say that the core thesis of Humeanism is that the laws are metaphysically explained by, and thus are reduced to, the Humean mosaic.

The strategy, then, is to say that the mosaic metaphysically explains the laws and the laws nomothetically explain the mosaic, thus avoiding circularity. This, in effect, was Loewer (2012) suggestion. Loewer, though, doesn't develop this strategy further. But further development is needed since it is just at this point that things start to get complicated.

To see why, notice that there are some nomothetic explanations which involve metaphysical explanations. Consider this case:

Interlevel Temperature: We are aiming to explain T, the fact that the temperature in this room now is 71 degrees. The explanation cites $P$, the state of the particles in this room and the surrounding area 10 minutes ago, and the fundamental laws. The way this this explanation works is that $\mathrm{P}$ and the laws together imply E, a fact about the kinetic energy of the particles in this room now. And, given the reduction of facts about temperature to facts about kinetic energy then $\mathrm{T}$ is nothing over and above E. 8

This is a nomothetic explanation of $\mathrm{T}$ from $\mathrm{P}$ and the laws. It is made up of a metaphysical explanation of $\mathrm{T}$ from $\mathrm{E}$ and a nomothetic explanation of $\mathrm{E}$ from $\mathrm{P}$ and the laws.

This case points to an important issue with the Loewer response: Certain metaphysical explanations can be part of larger nomothetic explanations, but the Loewer response relies on there being some metaphysical explanations that are not part of nomothetic explanations in this way. In particular, the metaphysical explanation of the laws from the mosaic cannot be part of nomothetic explanations. For

\footnotetext{
${ }^{8} \mathrm{I}$ also discuss this case in Bhogal (2017, section 2).
} 
example, the set of facts about the mosaic metaphysical explains the laws, and the laws nomothetically explain (at least partially) some particular fact about the mosaic F. But we can't chain together these explanations. If we did we would be left with the set of facts about the mosaic explaining a particular a particular fact about the mosaic F. But clearly this is unacceptably circular, a set of facts containing F cannot explain F.

So, for the Loewer response to be successful we need a reason why the metaphysical explanation of the laws from the mosaic cannot be part of nomothetic explanations, even when other metaphysical explanations can. To put it another way, we need a reason why we cannot chain this particular metaphysical explanation with nomothetic explanations. (So, the Loewer response needs to reject the 'Transitivity Condition' of Lange (2013) which implies that you can always chain metaphysical and nomothetic explanations. But more than this, it needs to reject chaining in this particular case. Miller (2015) and Hicks and van Elswyk (2015) provide some reasons to think that the Transitivity Condition fails, but we need more than this - we need an account of how and why this chaining can occur in some cases but not in the cases that would cause problems for Loewer-style responses to the circularity worry.)

The key to locating such a reason is to say more about the natures of nomothetic and metaphysical explanation. In particular we need to say more about the aims of such explanations - about what epistemic value those explanations yield.

Grasping an explanation of a fact seems to put us in an epistemically better position with respect to that fact - but what is this benefit? Let's look at the case of metaphysical explanation - I'm going to work with a very simple account of metaphysical explanation: A set of facts $S$ (wholly) metaphysically explains $\mathrm{F}$ if and only if $\mathrm{S}$ (wholly) grounds $\mathrm{F}$.

What is the epistemic value of such explanations? The natural answer, I take it, is that such explanations elucidate the underlying structure of the world. So, in grasping an explanation we gain knowledge of this structure - the grounding structure of the world. 
Perhaps if we didn't take talk of grounding, or related notions seriously, then we would have some other story about what the value of metaphysical explanation is. But I am taking such talk seriously. I'm assuming that there really is a grounding structure to the world in the way that people like Fine (2001), Schaffer (2009) and Rosen (2010) suggest. Given that there is this structure then it looks that the value of metaphysical explanations comes from limning this structure.

Anti-Humeans will likely think that nomothetic explanations work in a similar way. They think that the world comes with a built-in nomic structure, so it is reasonable to claim that the value of nomothetic explanations comes from elucidating this structure. For example, imagine that we explain the momentum of a particle by citing its collision with another particle and the laws governing that collision. In doing so we gain knowledge of the nomic structure of the world that gave rise to that event.

Things look different for Humeans though. It would be strange for them to think that the epistemic value of nomothetic explanations, like the particle-collision explanation, consists in elucidating nomic structure. Or, more carefully, the Humean should not think that elucidating nomic structure has substantial non-instrumental epistemic value. This is because, for the Humean, such nomic structure isn't a deep part of the world. Nomic structure, for the Humean, is defined up using a somewhat complicated systemization procedure. If the Humean thinks that knowledge of the output of the best system procedure is particularly epistemically important it has to be because it helps us with some other epistemic end. It is not plausible for the Humean to take knowledge of the output of this complex systemization procedure to have substantial non-instrumental epistemic value. If, on the other hand, there were deep, primitive governing laws, as certain anti-Humeans think, then it is much more plausible to take knowledge of these to have substantial non-instrumental epistemic value.

The Humean, then, should say something different about the epistemic value of nomothetic explanations. What they should do, I claim, is to appeal to an important strand of thinking in the Humean tradition, and in the philosophy of science more generally — a strand of thinking that stresses the value of unification. 
Stressing the importance of unification in science, and in explanation in particular, has a long tradition. Friedman (1974, p. 16), for example, gives an account of explanation in terms of unification, where we unify by 'reducing the total number of independent phenomena' that we have to accept. Kitcher (1981) gives an account in a similar spirit. But these accounts also locate themselves in a broader tradition, one that includes, for example, Kneale (1949, p. 92) who says that 'an explanation must in some sense simplify what we have to accept'. Similarly, Feigl (1970) claims that 'the aim of scientific explanation throughout the ages has been unification, i.e., the comprehending of a maximum of facts and regularities in terms of a minimum of theoretical concepts and assumptions'. And Hempel (1966) argues that 'what scientific explanation, especially theoretical explanation, aims at is... an objective kind of insight that is achieved by a systematic unification, by exhibiting the phenomena as manifestations of common, underlying structures and processes'

Further, unification seem to fit very naturally with the Humean picture. The mainstream Humean view on laws is the best system approach, where the laws are given by a systemization procedure - the laws are the facts that best balance simplicity and informative about the mosaic - that is extremely closely related to unification. And Loewer (1996, p. 113), in his discussion of the best system account, commits to the view that such laws explain via unification.

So, I claim that the Humean should say that the epistemic value of nomothetic explanations stems from unification. This suggestion seems very natural, and maybe even obvious. But surprisingly, Humeans have not committed to it in the literature, apart from the brief mention in Loewer (1996). Perhaps Humeans have always had this view in mind (though nothing Lewis, for example, said suggested that), or perhaps not. Either way, I'm going to commit to this view of the value of nomothetic explanation. And I'm going to understand unification in the Friedman way - we gain unification by reducing the number of phenomena that we need to accept independently. So, for example, the development of the kinetic theory of gases allowed us to give a single explanation of phenomena that we previously had to accept independently, like phenomenon to do with the relationship between the heat and pressure of 
gases, and to do with gaseous diffusion. In this way we reduce the number of phenomena that we need to accept independently.

Of course, there are questions about how to make this idea precise: How do we individuate phenomena? And what are the conditions under which phenomena can be accepted independently? But, given the large amount of ground that this paper covers, I can't discuss this in detail here. In any case, we won't be relying on fine judgements about unification - in the cases we consider the judgements about unification will be (hopefully) clear.

Let's look at an example. When we explain the momentum of a particle by citing the collision and the laws we see how the fact about the momentum fits into the most general patterns of the world because, for the Humean, the laws are the most general patterns of the world. In showing how this event fits into the general patterns of the world we are gaining unification by reducing the total number of independent phenomena that we have to accept. Instead of accepting all of the instances of collisions and the corresponding momentum changes separately we accept them together by identifying a general pattern. So, in explaining the fact of the particle's momentum using the collision and the laws we are gaining unification by assimilating the fact to this general pattern.

Again, the proposal is that the Humean should take the epistemic value of nomothetic explanation to consist in unification. Notice, though, that this does not force the Humean to accept the sorts of accounts of explanation that have been labeled 'unificationist' in the literature on explanation, for example, we don't have accept Friedman's (1974) account of explanation (even though we are working with his gloss on unification) or Kitcher's (1981) account. My suggestion is that the underlining epistemic aim of nomothetic explanation is unification. The surface-level account of explanation doesn't have to appeal to unification as long as the account, together with the Humean view of laws, validates this conception of the underlying aim.

The core idea, then, is that there is a difference, for the Humean, or at least a certain type of Humean, between the epistemic value of metaphysical and nomothetic explanations. Metaphysical explanation 
aims at elucidating underlying structure. Nomothetic explanation instead aims at unification. It is this divergence which is why some metaphysical explanations can be part of nomothetic explanations and some cannot.

In particular, explanations that contain as a part the explanation of the laws from the mosaic don't look like they will help achieve the aim of unification. In fact, it looks like they will significantly hinder it. Imagine that the facts about the mosaic metaphysically explain the laws, and the laws nomothetically explain (at least partially) a particular fact about the mosaic F. Chaining these together wouldn't leave us with a unificatory explanation - it wouldn't reduce the number of independent phenomena we have to accept. Rather, it tells us to understand a particular fact $\mathrm{F}$ by reference to an incredibly large number of distinct facts - all the facts about the mosaic.

Chaining the metaphysical explanation of the laws with a nomothetic explanation of any particular fact, then, will not yield explanations that help the cause of unification. 9

Here's a another way to think about why the metaphysical explanation of the laws from the mosaic doesn't help the cause of unification: When we unify we are trying to reduce the number of phenomena we accept independently by assimilating specific events to more general patterns. But the metaphysical explanation of the laws starts from the general patterns - the laws themselves - and reduces them to large numbers of specific facts - the facts about the mosaic. Clearly this procedure will not help unification. Any account of nomothetic explanation that fits with the aim being unification will not allow the metaphysical explanation of the laws from the mosaic to chain with any other nomothetic explanation.

On the other hand, some other metaphysical explanations can chain with nomothetic explanations because the resulting explanation would help the cause of unification. Interlevel Temperature is an example of this. The metaphysical explanations of facts about temperature in terms of facts about the

\footnotetext{
${ }^{9}$ The idea that it is the differing aim of scientific and metaphysical explanations that is the key to avoiding circularity for the Humean was independently developed by Dorst (ming) - though our stories about what those aims are are very different (Dorst has a conception of 'scientific explanation as fundamentally aimed at predictive utility' (p. 18)). But it's somewhat unclear what, for him, determines when a scientific explanation can chain with a metaphysical one and when it cannot.
} 
energy of particles helps us assimilate the facts about temperature to the more general patterns about the movement of particles. And thus such an explanation does help the cause of unification.

The thought that the reduction of facts about temperature to facts about energy helps the cause of unification is extremely common. (See, for example, Sklar (1993, p. 333), Oppenheim and Putnam (1958, p. 6), Friedman (1983, p. 239-241) among many others. 10 ) When we reduce facts about temperature to the facts about the energy of particles then the theory about energy can now capture all the phenomena about temperature because the facts about temperature are nothing over and above the facts about energy. We no longer need to accept facts about temperature independently of the facts about energy and so we reduce the number of phenomena that we need to accept independently.

To summarize, following Loewer, I claim that we avoid the initial circularity concern by distinguishing between metaphysical and nomothetic explanation - the facts about the mosaic metaphysically explain the laws, while the laws nomothetically explain facts about the mosaic. However, if we could chain these two explanations together we would reintroduce circularity. The reason we cannot chain these explanations, I suggest, is because there is a difference in the underlying value of metaphysical and nomothetic explanation. The ultimate epistemic value of nomothetic explanation is unification, while the ultimate epistemic value of metaphysical explanation is elucidating metaphysical dependence structure. We can't chain the metaphysical explanation of the laws with nomothetic explanations of particular facts to form a larger nomothetic explanation because doing so would hinder the cause of unification.

Or, to put it another way, scientists should ignore the reduction of laws to the mosaic when giving nomothetic explanations because it is not unificatory. But other metaphysical explanations - ones that do help the cause of unification — can be part of nomothetic explanations.

Again, the key idea here is the that the ultimate value of nomothetic explanation is unification, while the ultimate aim of metaphysical explanation is elucidating metaphysical dependence structure. I don't

\footnotetext{
${ }^{10}$ Though traditionally, the type of reduction considered in these contexts has not been metaphysical explanation, but rather a more linguistic conceptions of reduction. But the same ideas apply to metaphysical explanation.
} 
take these claims to be uncontroversial; they are significant commitments of my view. But they are commitments that are antecedently attractive to a certain type of Humean. As I noted above, the idea that the force of explanation in science comes from unification has a long tradition, and naturally flows from the Humean worldview. And the idea that the value of metaphysical explanation comes from elucidating metaphysical structure is a very natural one if we take that structure seriously. 11

\subsection{Two types of Fundamentality}

So, a Humean understanding of the value of nomothetic explanation, and how it differs from metaphysical explanation, allows us to respond to the explanatory circularity objection. Given this picture of the different aims it is reasonable for the Humean to think that metaphysical explanation diverges from the explanations given in science.

That response motivates a similar response to the non-fundamentality objection. The distinction between nomothetic and metaphysical explanation induces a distinction between nomothetic and metaphysical fundamentality. This is because there is a link between fundamentality and explanation: A fact is fundamental if and only if it is unexplained. The Humean takes the elements of the mosaic to be metaphysically unexplained. In doing so they take the elements of the mosaic to be metaphysically fundamental.12 The laws are metaphysically explained and thus metaphysically non-fundamental.

Analogously, I claim, something is nomothetically fundamental if and only if it is nomothetically unexplained. Some laws are nomothetically unexplained. Plausibly there are laws that are nomothetically explained by other laws, but some - like the most basic laws of physics - are not explained in this way. So, some laws are nomothetically fundamental.

Given our conception of nomothetic explanation as being tied to unification, the nomothetically fun-

\footnotetext{
${ }^{11}$ Notice that the Humean will not think that the aim of metaphysical explanation is also unification because, as we have seen, the metaphysical explanation of the laws from the mosaic is not unificatory. So, again, it is a commitment of my view that the aim of metaphysical explanation is illuminating metaphysical structure, not unification.

${ }^{12}$ This is generally accepted in the literature, though there is some disagreement, notably Fine (2001).
} 
damental is the unificatory base - it is the sparse set of facts from which we can unify all the other facts in the world. To say that a law is nomothetically fundamental, then, is to say that it is at the end point of unification - it is not one of the facts that we fit into a more general pattern, rather, it is the pattern that we fit facts into.

This, I think, is a natural way of interpreting claims about fundamentality of laws, when made in a scientific and not a metaphysical context. When scientists take a particular law to be fundamental they are not claiming that it is metaphysically fundamental - it's unlikely that they care at all about metaphysical fundamentality - rather they are saying that it is a fact that is taken as basic; we will not unify it with, or assimilate it to, any other facts. It's a fact that, in our unified understanding of the world, stands alone.

This, then, is the response to the non-fundamentality objection: The Humean can respect scientists' claims about the fundamentality of certain laws — interpreting their claims as about nomothetic fundamentality. There is a divergence between the scientific notion of fundamentality and the metaphysical notion.

\subsection{Non-supervenience and Two Types of Possibility}

I argued that distinguishing between two types of explanation could allow the Humean to respond to the circularity problem. Further, I suggested that we can extend this response to the non-fundamentality problem by distinguishing between two types of fundamentality. Similarly, I will argue distinguishing between metaphysical and nomothetic possibility is a promising way for the Humean to respond with the non-supervenience objection.

Or rather, they can respond to the the second version of the non-supervenience objection in this way. The first version of the objection is that the Humean says the wrong things about what is metaphysically possible - for example, that the Humean has to say that either the empty-GR or the empty-SR world 
is metaphysically impossible. The Humean just has to bite the bullet here and say, along with the Humeans we discussed in section 1.4, that our intuitions about metaphysical possibility are misleading in this case.

But the second version of the objection - that the Humean cannot make sense of the way that our scientific reasoning treats the cases like the empty-GR and empty-SR worlds as distinct - is the more pressing version. This is the version of the objection I'm considering. There are two steps to the response that I will suggest.

Firstly, I'll argue that in the sense of possibility relevant to scientific practice both the worlds are possible, even though at least one is metaphysically impossible. I call this sense of possibility nomothetic possibility. The Humean can say that supervenience does not hold in this space of possibility whilst it does hold in the space of metaphysical possibility. (The view remains distinctively Humean because the laws of nature are reduced to the Humean mosaic. ${ }^{13}$ The second step in this response is to say something about counterfactuals and explanations (and related notions) that allows us to understand how they can differ between these worlds.

But before that, in order to ward off confusion, it is very important to be clear that nomothetic possibility is not the same as the more familiar physical possibility. A proposition is physically possible relative to a world if it is consistent (in some sense) with the laws of that world. It is typically thought of as a restriction of metaphysical possibility. ${ }^{14}$ Nomothetic possibility, in my sense, does not have this structure.

So, central to this response is an appeal to a sense of possibility that is broader than metaphysical possibility — there are worlds that are metaphysically impossible but nomothetically possible. This is clearly

\footnotetext{
${ }^{13}$ Notice that responding to the second version of the non-supervenience objection - by saying that that the Humean should expect there to be a divergence between the metaphysical possibility and the possibilities countenanced in science also weakens the first version. After this response to the second version of the objection the opponent of Humeanism cannot defend their assertion that both the empty-GR and the empty-SR worlds are both metaphysically possible by appealing to the way that those possibilities are countenanced in scientific practice.

${ }^{14}$ Though in section 3.3 I will argue that the Humean should not think of it that way.
} 
a relatively unfamiliar idea. (Though appeals to senses of possibility that outrun metaphysical possibility are becoming more common (e.g. Jenkins and Nolan, 2012; Spencer, 2017).) Further, I claim that this sense of possibility is the relevant sense for scientific practice. So, we should start by considering why scientific practice would countenance possibilities that outrun the space of metaphysical possibility.

In general, I take it that the point of scientific reasoning about possibility is to explore the implications of scientific theories by looking at what alternative situations are consistent with the theory. Doing this can shed light on the theory we are considering but also it can shed light on the actual world. Let's look at a few examples of such scientific reasoning about possibility.

Imagine that the theory that we are investigating is Newtonian mechanics. We might, for example, investigate what the theory says about objects falling to earth. A natural place to start is by considering a situation where one object falls towards a much more massive object, and apart from those two objects, there is a vacuum. It is simple for us to use the theory to calculate what happens in this possibility. If we carry on this investigation we find variety of interesting things about what possibilities the theory allows. We find, for example, that in every situation where small objects fall towards earth in the absence of air resistance they accelerate at almost exactly the same rate. This sheds light on the theory. But also, exploring these possibilities - especially simple possibilities like worlds containing only two objects tells us a lot about how objects fall in the actual world. For example, by comparing actual cases of objects falling to these very simple situations we can see what the effect of air resistance is in our world. In particular, we find that air resistance is a lot more significant than we might have initially thought - it is the difference between the way a feather falls to the ground and the way a hammer does. More generally, scientific modeling often works like this - we look at simple cases which are consistent with the theory in question, seeing what happen in these cases is informative about the mechanics of the actual world.

Here's a very different example of scientific reasoning about possibility. When we explore the possibilities left open by non-inflationary Big Bang theory we find that nearly all of those possibilities lead 
to situations which are inconsistent with striking cosmological phenomena that we observe (like the uniform temperature of the microwave background radiation). Only a very specific choice of initial conditions allows the theory to be consistent with such phenomena (Maudlin, 2007, pp. 40-45). This is generally taken to be powerful evidence against the theory, and a key part of the motivation for the development of inflationary theories. Exploring the space of situations consistent with the theory, then, gives us reason to suspect that the theory is false.

Here's another case. Krauss (2012) argues that relativistic quantum field theory is inconsistent with stable situations where there are no particles. This is unlike, for example, standard general relativistic theories which are, as we noted, consistent with empty worlds. Plausibly, this has implications for what the explanation of the existence of particles is. 15 Seeing the differences between the theories that are consistent with there being no particles and those that are not is revealing both about the theories and about the nature of particles in our world.

All of this reasoning involves looking at what situations are logically consistent with particular scientific theories. This, I take it, is what scientific reasoning about possibilities ultimately consists in. Such reasoning can be enlightening in a variety of ways.

This suggests an understanding of what possibilities are relevant to scientific practice: they are those situations where the events in the world — i.e. the Humean mosaic — are logically consistent with the scientific theory. That is, where the Humean mosaic is consistent with the laws of that world and the patterns of explanations vindicated by those laws.

This might all seem pretty uncontroversial and obvious, but it has important implications. This is because the metaphysical explanation of the laws from the mosaic is not part of the scientific theory of the world. As we saw in section 2.1, scientific practice has a good reason to ignore such an explanation. And so the mosaic being consistent with the scientific theory of that world does not require that it is consistent with the scientific theory together with the metaphysical explanation of the laws from the

\footnotetext{
${ }^{15}$ Though it does not seem, contra Krauss, to have implications for why there is something rather than nothing.
} 
mosaic.

Consider an example. Assume that the best system of the world is given by Newtonian mechanics. So, on the Humean account we are working with, the correct scientific theory of the world is given by the laws of Newtonian mechanics. But the metaphysical explanation of the laws given in terms of facts about the mosaic is not part of the scientific theory - it is not part of Newtonian mechanics. Scientists ignore this metaphysical explanation (at least when doing science), and they are right to do so because this explanation runs contrary to the aim of unification.

So, when we consider what situations are consistent with Newtonian mechanics, we find that very simple situations like those we considered above - for example, the case where there is one body falling towards another much larger body, and those are the only two things in the world - are consistent with the theory. In fact, Newtonian mechanics has some precise predictions for how such a situation would unfold.

It is true that this situation is inconsistent with Newtonian mechanics and the metaphysical explanation of the laws from the mosaic because the best system for a world where those are the only two objects in the world would not output Newtonian mechanics. But this fact is irrelevant for scientific appeals to possibility. It would clearly be inappropriate for a philosopher to complain to a scientist that we shouldn't model objects falling by appealing to a two-object Newtonian world because such a world is metaphysically impossible. Again, the metaphysical explanation is not part of the scientific theory, and so it is not relevant for scientific judgments of possibility.

Similar reasoning applies to the empty-GR world. The empty mosaic is logically consistent with the theory of the world being given by general relativity, and thus the empty-GR world is a possibility that is relevant to scientific practice. This is true even though the empty mosaic is inconsistent with the theory of the world being given by the laws of GR together with the metaphysical explanation of the laws in terms of the mosaic. (And similarly with the empty-SR world.) 
We now have a picture of why scientific practice would countenance situations that are not metaphysically possible. The suggestion is that the metaphysical explanation of the laws from the mosaic is not part of the scientific theories considered by scientists, and there is a principled reason for this - such an explanation acts contrary to the goal of unification that is central to nomothetic explanation. Consequently, that metaphysical explanation does not constrain the possibilities that are relevant to scientific practice. 16

Let's give a name to this space of possibility that is relevant to science. Call it nomothetic possibility because it is importantly connected to nomothetic explanation. We have seen that the empty-GR world counts as nomothetically possible, even though it is metaphysically impossible (clearly the same reasoning applies to the empty-SR world).17

We should pause at this point and recap some important features of nomothetic possibility:

(1) The key feature of nomothetic possibility for our purposes is that the laws do not supervene upon the mosaic in the space of nomothetic possibility whilst they do in the space of metaphysical possibility. Again, the reason that laws supervene upon the mosaic in the space of metaphysical possibility is that the laws are metaphysically explained by the mosaic. But this explanation is ignored by scientific practice, because it hinders the cause of unification - and so is not relevant for what is nomothetically possible.

\footnotetext{
${ }^{16}$ The relation between scientific and metaphysical practice here is somewhat analogous to the relation between higher and lower-level sciences. Just as scientific practice countenances possibilities that are metaphysically impossible so higher-level sciences often countenance possibilities that are physically impossible. For example, economists often consider what their theories say about situations where information moves instantaneously and costlessly. The fact that such possibilities are physically impossible because they involve superluminal signaling does not stop them from being relevant for the practice of economics.

${ }^{17}$ Dasgupta (2018), in work defending relationalism about motion and about quantities like mass develops a strategy that is structurally very similar to the one implemented here. He distinguishes two senses of explanation and has that feed though to different senses of fundamentality and possibility. In fact, this general structure could apply to many cases where we might reasonably think that there is more than one type of explanation at work. As suggested in the previous footnote the relation between the higher and lower-level sciences is one possible case. Further, some metaethical views might want to distinguish two types of explanation. Some views reduce moral facts to some other facts, say facts about mental states or facts about our society. But defenders of those views might want to say that, nevertheless, certain facts are morally unexplained - they have no explanation from within our first-order moral theory, rather they are foundational to the theory. We could then distinguish different senses of fundamentality and possibility. In fact, I think this could be an attractive way to develop those metaethical views - having benefits that are closely analogous to the ones I describe for the Humean here.
} 
Rather, the nomothetic possibilities are the cases where the mosaic is logically consistent with the laws. This allows for non-supervenience in the space of nomothetic possibility.

(2) More specifically, both the empty-SR and empty-GR worlds are nomothetically possible, even though they are not metaphysically possible. The empty Minkowski spacetime is logically consistent with the laws of GR, so the empty-GR world is nomothetically possible. The empty Minkowski spacetime isnot logically consistent with the laws of GR and the metaphysical explanation of the laws in terms of the mosaic, so the empty-GR world is metaphysically impossible.

(3) So, some worlds are nomothetically possible but not metaphysically possible.

(4) Nomothetic possibility, for the Humean, in some ways mirrors what the non-Humean thinks is metaphysically possible. The non-Humean think that laws do not supervene on the mosaic in the space of metaphysical possibility and that things like the empty-SR and empty-GR worlds are possible because the mosaic is consistent with the relevant laws. My suggestion is that the Humean should think the analogous things about nomothetic possibility.

Of course, this isn't a full characterization of nomothetic possibility, there are a lot of questions still open. To pick just one, is it the case that everything that is metaphysically possible is nomothetically possible? Or are there certain metaphysical possibilities which just aren't relevant for the practice of science? There is a lot more work to be done on filling out the idea of nomothetic possibility. But hopefully we can start to see how such a notion could help with respect to the non-supervenience problem.

In fact, before we move on, we can motivate this sense of nomothetic possibility in a slightly different way as well, though it ultimately relies on the same ideas. The key is to consider the links between explanation and possibility. We can use the distinction between nomothetic and metaphysical explanation to motivate an analogous distinction in possibility - just as we leveraged the distinction between two types of explanation into a distinction between two types of fundamentality. 
There is a link between explanation and possibility - explanation has a distinctive modal signature.18 If A explains B then this has implications for how A and B can covary. More specifically, if A wholly explains B then B supervenes upon A.

The motivation for this is pretty simple: If I find a case where A is present but B is not then it looks like $\mathrm{A}$ is either wrong, or not complete, as an explanation of $\mathrm{B} .19$ This thought is common (though not totally uncontroversial) in the case of metaphysical explanation.20 And it also seems very intuitive when considering nomothetic explanations.

Different types of explanation imply different grades of supervenience though. Consider an analogy with moral explanation. Imagine that utilitarianism is true. The fact that an action A is wrong is wholly morally explained by its consequences for utility. The wrongness of the action will supervene upon the facts about utility then, but only in the range of worlds where utilitarianism is true. If we are considering worlds where utilitarianism is false we should not expect supervenience to hold across this range of worlds. The supervenience holds over the morally possible worlds. 21

Similarly, nomothetic explanation and metaphysical explanation imply supervenience over different spaces of worlds - nomothetic explanation constrains the nomothetically possible worlds; metaphysical explanation constrains the metaphysically possible worlds. More generally: If $A$ wholly explains $B$ then $B$ supervenes on $A$ in the corresponding space of possibility.

Metaphysical possibility is constrained by the metaphysical explanation of laws from the mosaic. Since the laws are metaphysically explained by the mosaic then the laws supervene on the mosaic in the space of metaphysically possible worlds. But, as we have seen, there is no nomothetic explanation of the laws

\footnotetext{
${ }^{18} \mathrm{I}$ 'm ignoring probabilistic explanations for now. In footnote 22 I'll note why this does not significantly affect my argument.

${ }^{19}$ deRosset (2010, section 4) explicitly defends such a principle, arguing that it is central to our practices of reasoning with, and about, explanations.

${ }^{20}$ Most notably, people who deny grounding necessitism (e.g. Leuenberger, 2014; Skiles, 2015) will reject this claim. Bennett (2017, section 3.3) discusses this in more detail, suggesting that our principle would need to be adapted slightly if we accept that there are background conditions required for $\mathrm{A}$ to explain B but which are not part of the explanation of $\mathrm{B}$.

${ }^{21}$ Which may or may not, depending on your view, overlap with the metaphysically possible worlds.
} 
from the mosaic. And so, there is no reason for there to be supervenience of the laws on the mosaic in the space of nomothetically possible worlds. Thus, the space of nomothetic possibility contains worlds which are not metaphysically possible.22

In particular, worlds like the empty-GR and empty-SR worlds are ruled out from both being metaphysically possible by the metaphysical explanation of the laws from the mosaic. But since there is no nomothetic explanation of the laws from the mosaic there is nothing to stop both of them being nomothetically possible.

Just as before, the central idea is that the metaphysical explanation of the laws from the mosaic is not relevant for what situations are taken as possible in scientific practice.23

So, we have the first step in the response to the non-supervenience problem. The empty-GR and emptySR worlds are not both metaphysically possible, but they are both possible in the sense relevant for scientific practice. The second step is to describe what is going on with counterfactuals and explanations (and related concepts) in these worlds.

The story here is very simple. Most accounts of explanations and counterfactuals (and other scientific modal notions, like causation) in the literature are given in terms of the mosaic and the laws. I can just appeal directly to such accounts. The reason that I have freedom to appeal to such accounts is because I countenance worlds, like the empty-GR world, where the laws and the mosaic are not connected via the Humean story about laws. Such worlds are metaphysically impossible, but they are nomothetically possible and important for the practice of science. The traditional Humean - one who does

\footnotetext{
${ }^{22}$ We can now see why ignoring the possibility of probabilistic explanation is not problematic. There are no probabilistic or indeterministic metaphysical explanations (see Bennett (2017, section 3.3.1)), and the key claim about nomothetic explanation is that there is no supervenience of laws on the mosaic in the space of nomothetic possibility. Clearly, countenancing indeterministic explanation will not allow us to recover any supervenience claim.

${ }^{23}$ Dasgupta's story about how two distinct types of possibility arise from two distinct types of explanation is superficially different, but, I think, rests on the same ideas. He takes the two different spaces of possibility to be generated via a recombination procedure from the two different fundamental bases. But, applying this to our setting, this relies on the idea that the metaphysical explanation of the laws from the mosaic constrains the ways in which the metaphysically fundamental can recombine to create metaphysical possibilities worlds, but it doesn't constrain the way that the nomothetically fundamental can recombine to create nomothetic possibilities.
} 
not countenance an important space of possibility that is broader than metaphysical possibility - has problems here because they ignore such worlds and so cannot appeal to these accounts. I do not face these problems.

Standard accounts of explanations and counterfactuals are going to have the result that there is a difference in the truth value of explanatory and counterfactual claims between the empty-GR world and the empty-SR world because there is a difference in the laws. So my view, being able to appeal to such accounts, will also accept that there is a difference in these respects between these nomothetically possible worlds.24

In summary, I suggest that the Humean should respond to the non-supervenience problem by saying that there is a divergence between the space of possibility relevant for scientific practice and metaphysical possibility - there is a space of nomothetically possible worlds that is broader than the space of metaphysically possible worlds. The Humean has reason to accept that supervenience of laws on the mosaic does not hold in this space of worlds. In particular, the empty-SR and empty-GR worlds are both possible in this sense. It's important to note that the view is still distinctively Humean, though, because the laws of nature are metaphysically explained by, and thus are nothing over and above, the Humean mosaic.

As I noted, there is still a lot more work to be done about the nature of nomothetic possibility if a fully satisfying Humean account along these lines is to be developed. But hopefully we have seen the outlines of how such a project might go.

\footnotetext{
${ }^{24}$ Perhaps you might worry that, given the introduction of nomothetic possibility and my argument that this sense of possibility plays a central role in science, metaphysical possibility becomes, in some sense, devalued; it no longer plays as central a role as it used to. I think there is some truth in this, but I don't think it's a bad result. For example, Sider, in his recent Locke Lectures, has discussed what he calls the 'postmodal' approach to philosophy, and to metaphysics in particular. This approach takes the central philosophical tools to be concepts like metaphysical explanation, or fundamentality, rather than modal notions. Like many philosophers, I am independently attracted to this approach. For example, I think that many philosophical positions - like physicalism, say - are best cashed out by appealing to metaphysical explanation rather than by appealing to modal notions. So I don't think it's problematic that my account here deemphasizes metaphysical possibility. Clearly there is much more to say about this, but equally clearly, this is not the right place to do that.
} 


\section{Nomothetic Possibility and Scientific Modalities}

I have sketched a new way of developing Humeanism, one that involves a divergence between elements of scientific and metaphysical practice. In particular, I distinguished nomothetic and metaphysical versions of explanation, fundamentality, and possibility and outlined how we might use these distinctions to deal with the circularity, non-fundamentality and non-supervenience problems.

In the last section I argued that the Humean should take there to be a divergence between the type of possibility appealed to in science and metaphysical possibility. I'm going to finish in this section by noting how this distinction between metaphysical and nomothetic possibility has consequences for other parts of scientific practice. In particular, I'll argue that countenancing nomothetic possibility allows the Humean to give accounts of various physical modalities - like chance, counterfactuals, and physical possibility - that are better than they could previously. The Humean can avoid problems that they face with these notions, and can do so in unified way - not by dealing with each in a piecemeal fashion.

\subsection{Counterfactuals}

Here is a problem that is closely related to the non-supervenience problem. The Humean appears to evaluate the truth of certain nested counterfactuals wrongly. Assume that the laws of GR hold in our world. Then consider the following counterfactual:

If the world were empty then (if there were a massive object in the world then spacetime would be curved)

This counterfactual is true. The rationale is fairly simple. If the world were empty then it would be the empty-GR world. But in any world where the laws are those of GR it's true that if there were massive objects then spacetime would be curved. 
But the traditional Humean fails to get this result. For them, if the world were empty then we would not be in a GR-world, we would be in a world where the laws were something much simpler. The laws of that world would almost certainly not associate masses with curvature of spacetime. And so in that world it is not true that if there was a massive object then spacetime would be curved, Lange (2009, p. 54), Sklar (2014, pp. 79-80) and Hall (2012, pp. 32-33) develop versions of this objection.25

Hall suggests that the Humean should respond by tweaking the standard possible world semantics for counterfactuals to get the desired result for the nested counterfactual. He suggests that in order to evaluate a counterfactual like if the world were empty then (if there were a massive object in the world then spacetime would be curved)' the procedure is as follows: The the first step is to find an empty world $w_{1}$ where the mosaic is a model of the actual laws. That is a world where the mosaic is consistent with the propositions that make up the actual laws, though not necessarily with the fact that those propositions are laws. Then we find the world $w_{2}$ closest to $w_{1}$ where the mosaic is again a model of the actual laws and there is a massive object in the world. If in $w_{2}$ spacetime is curved then the whole nested counterfactual is true.

It's not totally clear that this procedure gets the right result here - it depends on fine details of the closeness relation. But even assuming it does, there's a concern that this tweak to the semantics is somewhat ad hoc. In particular, the account in effect implies that we should evaluate a counterfactual differently when it is embedded in a larger counterfactual than when it is not. It's not clear why we should change the semantics in this way just to save Humeanism. 20

My account, on the other hand, gets the right result for the nested counterfactuals without such an ad hoc tweak to the semantics. All we need to do is to recognize that the relevant space of possibility is

\footnotetext{
${ }^{25}$ Hall (2015) is a much shorter version of the unpublished 2012 Hall paper mentioned above that doesn't include the content discussed here. Confusingly, though, both papers have the same title (though the longer, unpublished version is sometimes referred to as the 'Director's Cut') and it is the unpublished version that is more influential in the literature. My discussion here is of the unpublished 2012 paper.

${ }^{26}$ Loew and Jaag (2019) argue that a certain type of Humean does have motivation for accepting a semantics for counterfactuals such that so that the actual laws remain held fixed in nested counterfactuals. But their story doesn't motivate the Hall approach over mine, below.
} 
nomothetic possibility, not metaphysical possibility. Standard accounts of counterfactuals say that if the world were empty then the world would be an empty-GR world. And my account can appeal to those standard accounts because it accepts that there is an empty-GR world - a nomothetically possible one. The traditional Humean does not accept that there is a possible empty-GR world, and so had to deny that if the world were empty then it would be an empty-GR world - hence they fail to get the right result for the nested counterfactual.

So we have some reason to think that my account deals better with the nested counterfactual problem than traditional Humeans. But, there's a much more powerful reason for this to do with the way in which the nested counterfactual problems is part of a larger class of issues for the Humean. I'll describe this more soon.

\subsection{Chance}

Consider cases of 'undermining'. These are cases where a probabilistic law assigns a positive chance to a situation in which it is not a law.

It is often argued that the Humean has to accept the existence of such cases (Lewis, 1994; Thau, 1994; Arntzenius and Hall, 2003). Imagine, for example, that there is a probabilistic law L: coin tosses have a 50\% chance of landing heads. (Imagine too that coin tosses are irreducibly chancy.) Then consider a mosaic where every coin toss in the history of the world lands heads (and nothing about it violates any of the other laws). Assuming there are a finite number of coin tosses then the proposition P describing this mosaic will be assigned a positive probability.

But it is very plausible that if $\mathrm{P}$ is true then the Humean recipe for constructing laws from the mosaic will not have the result that $\mathrm{L}$ is a law. More likely, a law that assigns coin tosses a higher chance of landing heads would result.

It seems undeniable, though, that there is a positive chance of $\mathrm{P}$. This is puzzling, because it looks like 
the laws shouldn't assign a positive chance to propositions that are inconsistent with the laws.27 On one understanding this thought about consistency is in conflict with the case described — there is no metaphysically possible world where $\mathrm{L}$ is a law and $\mathrm{P}$ is true, yet $\mathrm{L}$ assigns $\mathrm{P}$ a positive probability.

However, there is a nomothetically possible world where $\mathrm{L}$ is a law and $\mathrm{P}$ holds. More generally, all cases of undermining involve laws assigning probabilities to situations which are models of the laws. That is, all cases of undermining involve the laws assigning probabilities to situations which are logically consistent with the laws (though not logically consistent with the laws along with the metaphysical explanation of the laws from the mosaic).

So, all cases of undermining involve laws assigning chances to (parts of) mosaics such that the law and the mosaic hold together in a nomothetically possible world. The Humean can, therefore, retain a thought about the laws not assigning a positive chance to situations which are impossible given the laws, as long as they understand it in terms of nomothetic, not metaphysical possibility.

More precisely, the Realization Principle of Schaffer (2007) (a strengthening of the Basic Chance Principle of Bigelow et al. (1993)) says that if proposition has a chance of more than zero, there must be a possible world where the history is the same as what it actually is, the laws are the same, and where the proposition is true. If we understand this principle as appealing to metaphysically possible worlds then the possibility of undermining cases shows that this principle rules out Humeanism about probabilistic laws. But the Humean can accept this principle on the understanding the relevant grade of possibility is nomothetic, not metaphysical.

In fact, the considerations in this section suggest something more. They suggest that chances are defined over (restrictions of) the set of nomothetically possible worlds, not metaphysically possible worlds; the algebra over which chance is defined is made up of nomothetically possible worlds. Consequently, chance doesn't always assign probability 1 to the metaphysically necessary facts. I think this is the right

\footnotetext{
${ }^{27}$ Much of the debate about undermining has been about what principles for how chance guides rational credence are plausible in the presence of undermining. I'm not going to consider this question here but I hope, in future work, to take on this question in light of what I say about undermining here.
} 
thing for the Humean to say about chance, though I don't have space to discuss it further here.

\subsection{Physical Possibility}

There are related issues with the traditional Humean account of physical possibility. Here are two natural, and commonly used, accounts of physical possibility (see, for example, Roberts (1998, p. 433), Schneider (2007, p. 312), Hall (2012, pp. 32-33)). The first account says that a proposition is physically possible relative to a world $w$ if and only if it is true in a (metaphysically possible) world that has the same laws as $w$. The second account says that a proposition is physically possible relative to a world $w$ if and only if it is true in a (metaphysically possible) world that is a model of the laws of $w$.

The traditional Humean move is to accept the second account (see again the authors cited above). The first account fails for the Humean because it implies that an empty mosaic is not physically possible relative to both GR and SR worlds. (Because for the empty mosaic to be physically possible relative to both GR and SR worlds there would have to be a metaphysically possible empty-GR world and a metaphysically possible empty-SR world.)

But nevertheless - as, I think, has gone unrecognized in the literature - this second account fails too. The reason it fails is because it construes physical possibility as a restriction of metaphysical possibility when, for the Humean, there are some propositions that are intuitively physically possible but not true in any metaphysically possible world.

Here is a case. Imagine the actual world is Newtonian. The following situation then seems physically possible: There are only three bodies in the world. Two bodies orbit a central body such that at all times a straight line connects the centers of mass of all the bodies. The outer bodies are the same mass and are always at the same distance from the central body. Call this setup S. The gravitational forces in this case are such that the outside bodies orbit whilst the central body does not ever move because it is always equally attracted by the two outside bodies. So, here is a causal claim, call it $\mathrm{C}$, that seems 
physically possible: $S$ holds and the distribution of the outside bodies causes the central body to remain motionless forever.

But $\mathrm{C}$ is, very plausibly, not true in any metaphysically possible world for the Humean. Because for $\mathrm{C}$ to be true not only must it be the case that the mosaic is the way that was described in the last paragraph, but it must also be the case that something like the laws of gravitation hold so that the central body is held in balance by the outside bodies. And it is implausible that any such law would follow from the best system account (or some alternative Humean story about laws) applied to such a mosaic. (Rather the laws of that mosaic would likely say that outside bodies orbit the middle, or something similar.) So $\mathrm{C}$ is not going to be judged to be physically possible on any account that involves a restriction of metaphysical possibility.

Rather than a restriction of metaphysical possibility what we need is an account where physical possibility is a restriction of nomothetic possibility. For example, consider an account which says that a proposition is physically possible relative to a world $w$ if and only if it is true in a nomothetically possible world that has the same laws as $w$. There is a nomothetically possible world in which the mosaic is as described above and the laws are Newtonian. And C is true in such a world (since we can appeal to standard accounts of causation to get this result). So such an account would evaluate the physical possibility of C correctly.

\subsection{A Class of Counterexamples}

In fact, this novel problem about physical possibility is an instance of a larger class of counterexamples to Humeanism, one which subsumes the problems about nested counterfactuals and undermining discussed above. Any time there are claims that hold in virtue of the mosaic and the laws of a different world then the traditional Humean is prone to evaluate such claims wrongly (because of the restrictions the traditional Humean has on how mosaics can combine with laws). 
The claim about the physical possibility of $\mathrm{C}$ is an example of this - the physical possibility of the causal claim C holds in virtue of the mosaic and the laws at other worlds.

The nested counterfactual objection has the same structure. The nested counterfactual we considered was: If the world were empty then (if there were a massive object in the world then spacetime would be curved). The truth of this depends upon the laws of another world. We evaluate this counterfactual by looking at the closest empty world and then seeing what the laws are in that world. If the laws are those of General Relativity, then it is true that if there were a massive object in the world then spacetime would be curved, and so the whole nested counterfactual is true. But, of course, the Humean will not think that the laws of GR hold in any empty world. They will take the laws to be much simpler, thus the counterfactual in the consequent would be false, and the whole nested counterfactual is false.

So, the nested counterfactual holds in virtue of the mosaic and the laws of a different possible world. In this case, the closest empty world. The Humean evaluates this proposition wrongly because of the restrictions it puts on how laws can combine with mosaics.

The non-supervenience objection is also of this kind. Consider the proposition that it is possible that there is an empty-GR world and an empty-SR world. This proposition seems to be true, but the Humean incorrectly evaluates it because it depends on the laws, as well as the mosaics, of other possible worlds.

Looking at these cases we have almost a recipe for generating counterexamples to Humeanism. Take a proposition that has a component that pushes us to look at another possible world, and a component that relies upon the laws as well as the mosaic of that different world. Put these components together and we have a proposition that holds in virtue of the mosaic and the laws of a different possible world - the traditional Humean will misevaluate some of these propositions.

So, for example, we can easily, by slightly adapting the cases we have already seen, generate counterexamples to traditional Humeanism where the Humean misevaluates certain counterfactual claims about 
explanations; counterfactual claims about chances; claims about the physical possibility of chances, claims about the chances of chances, claims about the physical possibility of laws, and so on. I'll leave it to the reader to generate these, and other, counterexamples.

The point of all this, is that since there is this unified class of counterexamples to Humeanism, responding in a piecemeal way to the counterexamples - for example, by tweaking the semantics of counterfactuals to deal with the nested counterfactual objection, is unsatisfactory. The Humean needs a unified response. And the appeal to nomothetic possibility provides this response - in the space of nomothetically possible worlds there are looser restrictions on how mosaics can combine with laws and so counterexamples cannot be generated in the same way. Expanding the relevant space of worlds from metaphysical to nomothetic defuses this class of counterexamples.

The fact that it allows this unified response to a variety of counterexamples is perhaps the most powerful reason for the Humean to countenance nomothetic possibility.

\section{Conclusion}

I have outlined a new way of developing Humeanism - one that develops Loewer's response to the circularity problem and extends it in order to deal with the other problems that Humeans face. This way of developing Humeanism points towards certain novel responses to the non-fundamentality and nonsupervenience problems. In particular, recognizing that there are important types of fundamentality and possibility that are distinct from metaphysical fundamentality and possibility allows us to respond to the non-fundamentality and non-supervenience problems. Further, this new grade of possibility seems to give the Humean certain advantages with respect to their accounts of counterfactuals, chance and physical possibility.

This picture is motivated by the idea that the aims of science diverge from the aims of metaphysics. While metaphysicians want to understand the deep dependence structure of the world the aim, or at 
least one aim, of science is to unify. This warrants a certain kind of divergence between the practice of metaphysics and that of science - the aim of unification means that it is not part of the practice of scientific explanation to reduce the laws to anything else (and this is true even if particular scientists, in their philosophical moments, were to accept Humeanism). This feeds through to the other elements of the practice of science - like the possibilities that scientists countenance diverging from metaphysical possibility. The divergence between metaphysical and scientific practice, that the objections to Humeanism make salient, is not something that that refutes Humeanism. Rather it is something that follows from a natural Humean understanding of science.

Of course, a lot more work is required in order to fully flesh this out. What I have done is just provide the outline for a larger Humean project. But I think that the picture outlined here is promising.

One final point: I expect a certain type of reaction from a totally committed anti-Humean. They will think their view fits better with scientific practice and will consider the view developed in this paper as ad hoc or overly complicated machinery that is supposed to replicate what their view gets naturally. One part of this is right. Often anti-Humeans build their metaphysics in order to mirror scientific practice - Maudlin, for example, is pretty explicit about this approach. And if the anti-Humean builds their metaphysics in order to mirror scientific practice then anyone who wants to capture the practice will replicate, in some sense, elements of the anti-Humean view. For people who see no attraction at all in the Humean picture this will be enough to reject the view. But this paper is not for them. For people who find some attraction in the austerity and elegance of the Humean picture then there is value in the account developed here - it allows you to enjoy those attractions whilst avoiding unpalatable consequences. 


\section{References}

Armstrong, D. M. (1983). What is a Law of Nature. Cambridge Studies in Philosophy. Cambridge: Cambridge University Press.

Arntzenius, F. and N. Hall (2003). On What We Know about Chance. The British Journal for the Philosophy of Science 54(2), 171-179.

Beebee, H. (2000). The Non-Governing Conception of Laws of Nature. Philosophy and Phenomenological Research 61, 571-594.

Bennett, K. (2017). Making Things Up. Oxford University Press.

Bhogal, H. (2017). Minimal Anti-Humeanism. Australasian Journal of Philosophy 95(3), 447-460.

Bhogal, H. and Z. R. Perry (2017). What the Humean Should Say About Entanglement. Noûs 51(1), 74-94.

Bigelow, J., J. Collins, and R. Pargetter (1993). The Big Bad Bug: What Are the Humean's Chances? British Journal for the Philosophy of Science 44(3), 443-462.

Bird, A. (2007). Nature's Metaphysics. OUP.

Carroll, J. (1994). Laws of Nature. Cambridge University Press.

Dasgupta, S. (2018). How to be a Relationalist.

deRosset, L. (2010). Getting Priority Straight. Philosophical Studies 149(1), 73-97.

Dorst, C. (forthcoming). Humean Laws, Explanatory Circularity, and the Aim of Scientific Explanation. Philosophical Studies, 1-23. 
Earman, J. and J. T. Roberts (2005). Contact with the Nomic: A Challenge for Deniers of Humean Supervenience About Laws of Nature Part I: Humean Supervenience. Philosophy and Phenomenological Research 71(1), 1-22.

Esfeld, M., D. Lazarovici, M. Hubert, and D. Dürr (2014). The Ontology of Bohmian Mechanics. British Journal for the Philosophy of Science 65(4), 773-796.

Feigl, H. (1970). The “Orthodox” View of Theories: Remarks in Defense as Well as Critique. Minnesota Studies in the Philosophy of Science.

Fine, K. (2001). The Question of Realism. Philosophers' Imprint.

Friedman, M. (1974). Explanation and Scientific Understanding. The Journal of Philosophy 71(1), $5-19$.

Friedman, M. (1983). Foundations of Space-Time Theories. Princeton: Princeton University Press.

Hajek, A. (2010). Mises Redux-Redux : Fifteen Arguments Against Finite Frequentism. In Philosophy of Probability: Contemporary Readings, pp. 209-227. Routledge.

Hall, N. (2012). Humean Reductionism About Laws Of Nature. Unpublished manuscript.

Hall, N. (2015). Humean reductionism about laws of nature. In B. Loewer and J. Schaffer (Eds.), $A$ Companion to David Lewis, Chapter 17, pp. 262-277. Wiley.

Hempel, C. G. (1966). Philosophy of Natural Science. Englewood Cliffs, N.J.,Prentice-Hall.

Hicks, M. T. (ms.). What the Laws (Can't) Explain.

Hicks, M. T. and P. van Elswyk (2015). Humean Laws and Circular Explanation. Philosophical Studies 172, 433-443.

Jenkins, C. S. and D. Nolan (2012). Disposition impossible. Noûs 46(4), 732-753. 
Kitcher, P. (1981). Explanatory unification. Philosophy of Science 48(4), 507-531.

Kneale, W. (1949). Probability and Induction. OUP.

Krauss, L. (2012). A Universe from Nothing: Why There Is Something Rather than Nothing. Atria Books.

Lange, M. (2009). Laws and Lawmakers: Science, Metaphysics, and the Laws of Nature. Oxford University Press.

Lange, M. (2013). Grounding, scientific explanation, and Humean laws. Philosophical Studies 164, $255-261$.

Lange, M. (2018). Transitivity, Self-Explanation, and the Explanatory Circularity Argument Against Humean Accounts of Natural Law. Synthese 195(3), 1337-1353.

Leuenberger, S. (2014). Grounding and Necessity. Inquiry 57(2), 151-174.

Lewis, D. (1983). Philosophical Papers, Volume 1. Philosophical Papers, Volume 1. Oxford: Oxford University Press.

Lewis, D. (1994). Humean Supervenience Debugged. Mind 103(412), 473-490.

Loew, C. and S. Jaag (2019). Humean Laws and (Nested) Counterfactuals. The Philosophical Quarterly.

Loewer, B. (1996). Humean supervenience. Philosophical Topics 24(1), 101-127.

Loewer, B. (2012). Two accounts of laws and time. Philosophical Studies 160(1), 115-137.

Maudlin, T. (2007). The Metaphysics Within Physics. The Metaphysics Within Physics. New York: Oxford University Press.

Miller, E. (2014). Quantum entanglement, bohmian mechanics, and humean supervenience. Australasian Journal of Philosophy 92(3), 567-583. 
Miller, E. (2015). Humean Scientific Explanation. Philosophical Studies 172(5), 1311-1332.

Oppenheim, P. and H. Putnam (1958). Unity of Science as a Working Hypothesis. Volume 2. University of Minnesota Press.

Roberts, J. (1998). Lewis, Carroll, and Seeing Through the Looking Glass. Australasian Journal of Philosophy 76(3), 426-438.

Roberts, J. T. (2008). The Law-Governed Universe. Oxford University Press.

Rosen, G. (2010). Metaphysical Dependence: Grounding and Reduction. In Bob Hale and Aviv Hoffmann (Eds.), Modality: Metaphysics, Logic, and Epistemology, pp. 109-36. Oxford University Press.

Schaffer, J. (2007). Deterministic Chance? British Journal for the Philosophy of Science 58(2), 113-140.

Schaffer, J. (2009). On What Grounds What. In D. Manley, D. J. Chalmers, and R. Wasserman (Eds.), Metametaphysics: New Essays on the Foundations of Ontology. OUP.

Schaffer, J. (2015). What Not to Multiply Without Necessity. Australasian Journal of Philosophy 93(4), 644-664.

Schneider, S. (2007). What is the Significance of the Intuition That Laws of Nature Govern? Australasian Journal of Philosophy 85(2), 307-324.

Skiles, A. (2015). Against Grounding Necessitarianism. Erkenntnis 80(4), 717-751.

Sklar, L. (1993). Physics and Chance: Philosophical Issues in the Foundations of Statistical Mechanics. Cambridge University Press.

Sklar, L. (2014). Physical Theory: Method and Interpretation. Oxford Univerity Press.

Skow, B. (2016). Reasons Why. Oxford University Press UK. 
Spencer, J. (2017). Able to do the Impossible. Mind 126(502), 466-497.

Thau, M. (1994). Undermining and admissibility. Mind 103(412), 491-504.

Tooley, M. (1977). The Nature of Laws. Canadian Journal of Philosophy 7(4), 667-698. 